\title{
A Study of Correlation between Subjective Sensation of Nose Block and Objective Assessment of Nasal Patency by Cold Spatula Test in a Patient with Nose Block
}

\author{
${ }^{1}$ Parth B Kapadia, ${ }^{2}$ Anand V Chaudhari, ${ }^{3}$ Jaymin A Contractor, ${ }^{4}$ Rahul B Patel, ${ }^{5}$ Jay N Suratwala, ${ }^{6}$ Jalpa A Patel
}

\begin{abstract}
The complaint of a nose block is often a complex clinical problem by itself. There is no consensus about the value of objective measurements of nasal patency. However, the perception of nasal airflow ultimately is a subjective sensation and, therefore, difficult to quantify. In this study, we have objectively evaluated 75 patients coming to our institute with the complaint of nose block. The mean area of air blast of both the nasal cavities of each patient was calculated by cold spatula test using modified "Glatzel mirror (GM)," and the subjective sensation of nose block was compared on each visit. We observed that both medical and surgical interventions objectively did improve the area of air blast in both unilateral and bilateral nose block patients. This also correlated with the increase in subjective sensation of relief from nose block in the patients. Thus, we could conclude that in patients with nose block, both medical and surgical interventions improved the area of air blast $(p<0.001)$, which correlated with the subjective sensation of relief in nose block. Thus, in standard conditions, cold spatula test using modified GM can be used to objectively evaluate the nasal patency.
\end{abstract}

Keywords: Cold spatula test, Glatzel mirror, Nasal patency, Nose block.

How to cite this article: Kapadia PB, Chaudhari AV, Contractor JA, Patel RB, Suratwala JN, Patel JA. A Study of Correlation between Subjective Sensation of Nose Block and Objective Assessment of Nasal Patency by Cold Spatula Test in a Patient with Nose Block. Clin Rhinol An Int J 2017;10(1):1-5.

Source of support: Nil

Conflict of interest: None

\section{INTRODUCTION}

The complaint of a blocked nose is often a complex clinical problem involving mucosal, structural, and even

\footnotetext{
${ }^{1,5}$ Senior Resident, ${ }^{2,4}$ Assistant Professor, ${ }^{3}$ Professor and Head ${ }^{6}$ Ex-Senior Resident

1,2,4-6 Department of Otorhinolaryngology, Head and Neck Surgery, Government Medical College and New Civil Hospital Surat, Gujarat, India

${ }^{3}$ Department of ENT and Head and Neck Surgery, Government Medical College and New Civil Hospital, Surat, Gujarat, India

Corresponding Author: Jaymin A Contractor, Professor and Head, Department of ENT and Head and Neck Surgery Government Medical College and New Civil Hospital, Surat Gujarat, India, Phone: +912612778089, e-mail: dr_jcontractor@ yahoo.co.in
}

psychological factors. Due to nasal cycle, the airflow in each nostril may be different; hence, it is difficult to correlate with the objective measurement of nasal patency in a normal individual. The perception of nasal airflow ultimately is a subjective sensation of nasal patency, which is most of the time unnoticed by a person unless he or she develops sensation of nasal block as a symptom. Our research questions are: (1) Whether the symptoms of nose block really indicate actual obstruction of air flow irrespective of nasal cycle; and (2) whether the symptom of nose block indicates obstructive pathology in nose. Even so, efforts are continuously being made to improve our ability to "objectively" measure nasal patency. The gold standard would be a quantifiable, reproducible, objective test with a strong correlation to the subjective perception of nasal airflow. Such a test would help us in diagnosing the degree and probably the cause of nasal obstruction. It would also be useful for evaluating the results of surgical interventions aimed at improving nasal patency. Use of the Glatzel mirror (GM) in a cold spatula test is an old and simple technique for objectively and momentarily verifying the nasal patency. The clinical examiner may obtain a momentary assessment of nasal patency by comparing the condensation area of each nasal fossa. ${ }^{1}$ In this study, we have evaluated the current status of our ability to measure nasal patency objectively and its correlation with the subjective sensation of nose block.

\section{AIMS AND OBJECTIVES}

- To correlate the subjective sensation of nose block with the results of cold spatula test

- To study the usefulness of cold spatula test in investigating cases of nose block

- To analyze the results of cold spatula test in relation to the result of treatment in terms of improvement in symptom of nose block

\section{MATERIALS AND METHODS}

\section{Inclusion Criteria}

- All patients with c/o nasal blockage

- Age $\geq 6$ years

- Sex: $M / F$ 


\section{Exclusion Criteria}

- Age $<6$ years

- External nasal deformity

- Patients/guardians refusing to give consent for study

- Patients who fail to come for regular follow-up

- Patients with poor general medical condition

This was a prospective cohort observational study conducted in 75 patients of age $\geq 6$ years who presented in the ear-nose-throat outpatient department (OPD) with the complaint of nose block. All the patients underwent detailed evaluation by history, OPD examination including anterior rhinoscopy, nasal endoscopy for the decision of further medical and/or medical management as per departmental protocol.

The primary investigator was kept blind from the findings of anterior rhinoscopy and nasal endoscopy during cold spatula test to avoid bias. The participants were asked to refrain from smoking and topical or systemic nasal decongestant drugs on the day of the cold spatula test. Three reliable recordings of area of fogging were noted and the average counted as a final area of air blast to minimize the variables.

Nasal patency was objectively assessed by calculating the air blast in $\mathrm{mm}^{2}$ by the cold spatula (modified GM) for complaint of nasal blockage, before and after definitive treatment. For calculation in our study, percentage of feeling of nasal blockage was taken into account to compare and correlate with area of fogging of air blast. The temperature $\left(25-35^{\circ} \mathrm{C}\right)$ and relative air humidity $(50-65 \%)$ of the test site were kept constant throughout testing. The following protocols were applied:

- Acclimatizing - participants remained seated for 5 minutes in the test environment.

- Patients were asked to rate the feeling of sensation of nasal blockage in percentage.

- Patients breathed on the modified GM. Modified GM is a cold metallic spatula with shiny surface. This mirror

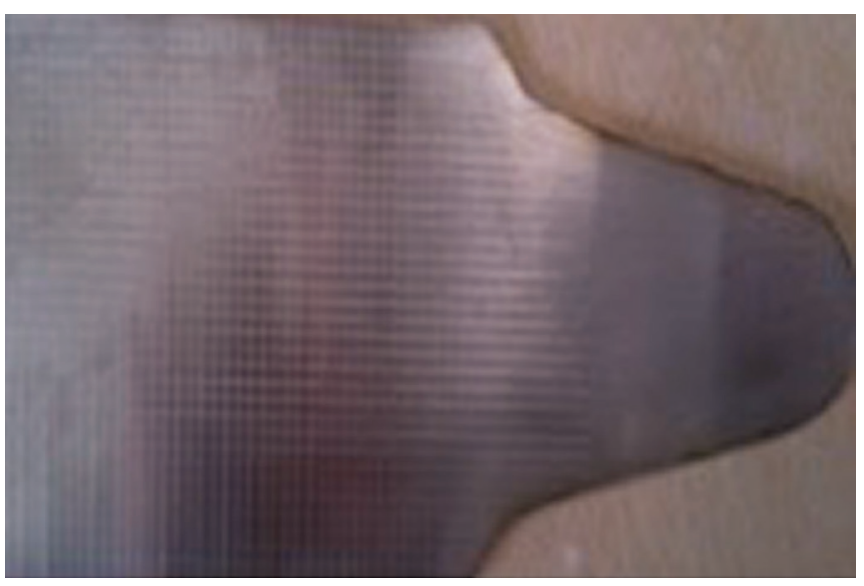

Fig. 1: Modified GM (cold spatula) was marked permanently with a calibrated millimeter scale of $2 \mathrm{~mm}$, and then it was used for an objective assessment of nasal patency. Mirror measurements were done with seated subjects and the head in the orthostatic position. The mirror was placed horizontally under the nostrils of participants with the mirror's zero point at a middistance from a point under the columella and philtrum. Participants were asked to breathe in a natural spontaneous manner through both nostrils with no extra inspiratory or expiratory effort and keep the mouth and eyes closed.

The condensation of air blast was marked with an overhead transparency-marking pen on the mirror. The condensation contour was measured along the greater and lesser axes, on the left and right sides separately. The greater axis was obtained starting from the central point (zero mark) until the longest dimension (within the contour). The lesser axis was also selected at $90^{\circ}$ to longer axis. From the greater and lesser axes, area of air blast was calculated in $\mathrm{mm}^{2}$ (Figs 1 and 2).

\section{OBSERVATIONS AND RESULTS}

Modified GM was used as a cold spatula. Measurements of area of air blast on cold spatula of right and left nostrils were done on each visit, both before and after treatment (medical and/or surgical). Totally, 75 patients participated in our study. All of these patients underwent surgical intervention with or without prior medical management. Mean age of the patients was $25.6 \pm 11.5$ years with male predominance. Majority of the patients (73\%) had unilateral nose block and among them, 38\% had complaint of left nose block. About 35\% of the patients had deviated nasal septum (DNS) to the left as evident in nasal endoscopy. With bilateral nose block, $85 \%$ patients had mouth breathing.

We have analyzed the correlation between the subjective sensation of nose block (including unilateral and

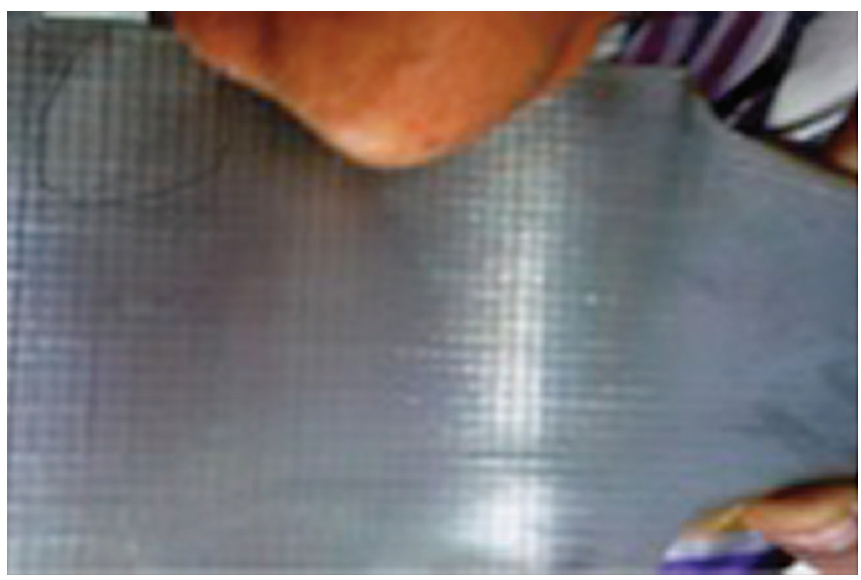

Fig. 2: Area of right nasal air blast marked 
Table 1: Correlation between subjective sensation of nose block with the air blast measured by cold spatula test during various follow up visits

\begin{tabular}{lllll}
\hline & Visits & $\begin{array}{l}\text { Mean left air } \\
\text { blast in left nose } \\
\text { block }\left(\mathrm{mm}^{2}\right)\end{array}$ & $\begin{array}{l}\text { Mean right air } \\
\text { blast in right nose } \\
\text { block }\left(\mathrm{mm}^{2}\right)\end{array}$ & $\begin{array}{l}\text { Mean bilateral air } \\
\text { blast in total nose } \\
\text { block }\left(\mathrm{mm}^{2}\right)\end{array}$ \\
\hline Pretreatment & 0 & 78.7 & 83.8 & 115.8 \\
After medical management & 1 & 121.8 & 148.2 & 232.4 \\
After surgical management & 2 & 134.4 & 145.4 & 215.1 \\
& 3 & 209.4 & 204.6 & 390.5 \\
& 4 & 280 & 288 & 588.2 \\
\hline
\end{tabular}

bilateral) with the air blast measured by cold spatula test. First visit has been considered as visit 0 . Subsequent visits were numbered from visit 1 to 5 . This includes visits 2 and 3 during medical treatment if given and postsurgical visits 3, 4, and 5 (Table 1 ).

The subjective sensation of nose block on the first visit was considered to be $100 \%$. For this, all the patients were divided into following four groups according to the subjective sensation of nose block documented by the patient in percentage out of 100: Group I: $<25 \%$, group II: $\geq 25 \%$ to $<50 \%$, group III: $\geq 50 \%$ to $<75 \%$, group IV: $\geq 75 \%$. Observation shows that all 29 (100\%) patients with left side nose block were in group IV, i.e., having $\geq 75 \%$ nose block on visit 1 . On visit 5, patients numbering $21(72.4 \%)$ fall in group I, i.e., having $<25 \%$ nose block and $8(27.6 \%)$ patients in group II, i.e., $\geq 25 \%$ to $<50 \%$. Observations for right nose block show that $22(84.6 \%)$ patients were in group IV, i.e., $\geq 75 \%$ nose block and $4(15.4 \%)$ patients were in group III, i.e., $\geq 50 \%$ nose block on visit 1 . On visit 5 , patients numbering $22(84.6 \%)$ fall in group I, i.e., having $<25 \%$ nose block and $4(15.4 \%)$ patients in group II, i.e., $\geq 25 \%$ to $<50 \%$. Observations for bilateral nose block show that 16 (80\%) patients had $\geq 75 \%$ (group IV) nose block and $4(20 \%)$ patients had $\geq 50 \%$ (group III) nose block on visit 1 . On visit 5, patients numbering 20 (100\%) fall in group I, i.e., having <25\% nose block (Table 2 ).

On each subsequent visit (including those during medical treatment, i.e., visit 2, and postsurgical treatment, i.e., visits 3, 4, and 5), there was a gradual increase in percentage of patients from group III to group I. Postsurgically, none of the patients was found in group IV. Also, the mean unilateral air blast along with the mean total air blast has increased on each subsequent visit. The analysis of variance (ANOVA) test had detected that the difference in the mean air blast between different visits and different groups was statistically significant. In our study, it was observed that patients had positive findings of anatomical obstruction in nose on diagnostic nasal endoscopy (DNE) at the side of nose block (Table 3).

\section{DISCUSSION}

Totally 75 patients $(n=75)$ participated in our study. All patients in the study underwent surgical intervention with or without prior medical management. About $65.3 \%$ received presurgical medical management. The medical management comprised topical decongestants, topical

Table 2: Correlation between nose block and air blast

\begin{tabular}{|c|c|c|c|c|c|}
\hline \multirow{2}{*}{$\begin{array}{l}\text { Groups (as per } \\
\text { percentage of } \\
\text { nose block) }\end{array}$} & \multicolumn{5}{|c|}{ No. of patients } \\
\hline & Visit 1 & Visit 2 & Visit 3 & Visit 4 & Visit 5 \\
\hline \multicolumn{6}{|c|}{ Correlation between left nose block $(n=29)$ and left air blast } \\
\hline I & $0(0 \%)$ & $0(0 \%)$ & $2(6.9 \%)$ & $11(37.9 \%)$ & $21(72.4 \%)$ \\
\hline II & $0(0 \%)$ & $1(3.5 \%)$ & $10(34.5 \%)$ & $16(55.2 \%)$ & $8(27.6 \%)$ \\
\hline III & $0(0 \%)$ & $5(17.2 \%)$ & $17(58.6 \%)$ & $2(6.9 \%)$ & $0(0 \%)$ \\
\hline IV & $29(100 \%)$ & $23(79.3 \%)$ & $0(0 \%)$ & $0(0 \%)$ & $0(0 \%)$ \\
\hline \multicolumn{6}{|c|}{ Correlation between right nose block $(n=26)$ and right air blast } \\
\hline I & $0(0 \%)$ & $0(0 \%)$ & $0(0 \%)$ & $11(42.3 \%)$ & $22(84.6 \%)$ \\
\hline II & $0(0 \%)$ & $1(3.9 \%)$ & $14(53.8 \%)$ & $13(50 \%)$ & $4(15.4 \%)$ \\
\hline III & $4(15.4 \%)$ & $5(19.2 \%)$ & $12(46.2 \%)$ & $2(7.7 \%)$ & $0(0 \%)$ \\
\hline IV & $22(84.6 \%)$ & $20(76.9 \%)$ & $0(0 \%)$ & $0(0 \%)$ & $0(0 \%)$ \\
\hline \multicolumn{6}{|c|}{ Correlation between bilateral nose block $(n=20)$ and total air blast } \\
\hline I & 0 & $0(0 \%)$ & $1(5 \%)$ & $7(35 \%)$ & $20(100 \%)$ \\
\hline II & 0 & $0(0 \%)$ & $11(55 \%)$ & $13(65 \%)$ & $0(0 \%)$ \\
\hline III & $4(20 \%)$ & 7 (35\%) & $8(40 \%)$ & $0(0 \%)$ & $0(0 \%)$ \\
\hline IV & $16(80 \%)$ & $13(65 \%)$ & $0(0 \%)$ & $0(0 \%)$ & $0(0 \%)$ \\
\hline
\end{tabular}


Table 3: Distribution of patients according to nasal endoscopy findings

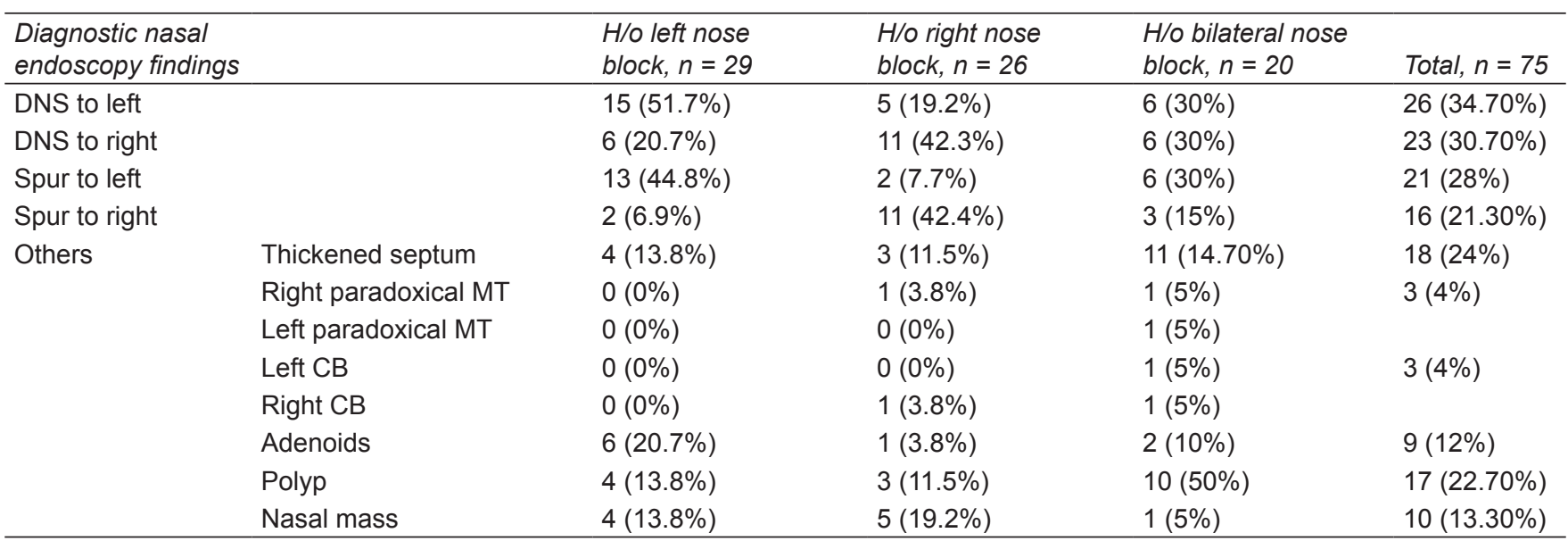

MT: Middle turbinate; CB: Concha bullosa; DNS: Deviated nasal septum

steroids, oral antihistamines, and/or oral steroids. On determining outcome of medical and surgical management in terms of subjective sensation in relief of nose block, it is observed that posttreatment, both interventions objectively improve the area of air blast irrespective of laterality of the nose block. It was observed that air blast increased significantly after surgical management in comparison with that of medical management, which was correlated with the subjective improvement in bilateral nose block. This was in concordance with the study done by Hox et al, ${ }^{2}$ which states that "Visual Analogue Scale scores for nasal blockage correlated well with Peak Nasal Inspiratory Flow values." Melo et al ${ }^{3}$ did a study on mouth breathing evaluation by use of GM and peak nasal inspiratory flow. Their aim was to compare the use of the GM and peak nasal inspiratory flow in the evaluation of mouth-breathing participants. The GM was reliable in identifying participants with and without nasal obstruction.

All data proving this observation were statistically significant (ANOVA, $p<0.001)$. This means that medical as well as surgical treatment resulted in great benefit to the patient in relation to his/her subjective sensation of nose block. Cold spatula test has also shown the increase in the mean area of air blast on each subsequent visit, thus correlating well with the subjective sensation of the nose block; it is statistically significant. This was in concordance with the study by Gulati et al. ${ }^{4}$ In this study, rhinomanometric improvement in nasal airflow and decreased nasal resistance were found in $88 \%$ patients after surgery.

Brescovici and Roithmann ${ }^{1}$ did a study on modified GM test reproducibility in the evaluation of nasal patency. From their study, they concluded that there is no significant correlation between the subjective perception of breathing and the condensation area. However, this study was done in normal individuals without symptom of nose block, so subjective perception of breathing is minimal and variable due to nasal cycle. However, in our study, it was observed that patients had positive findings of anatomical obstruction in nose on DNE at the side of nose block (Table 3). This was an indirect correlation with the nasal air blast and obstructive nasal findings, which might contribute for symptom of nose block. After correction of obstructive nasal findings, nasal air blast and symptom of nose block were significantly improved. These suggest that in symptomatic patients when the nasal air blast is diminished, there are fair chances of positive findings of anatomical obstruction including DNS, spur, concha bullosa, polyp, mass, etc. This was in concordance with the study by $\mathrm{Lio}^{5}$ and Dadgarnia et $\mathrm{al}^{6}$. In these studies, researchers observed an improvement in resistance of the cross-sectional area on the narrower side after septoplasty, while no variations were witnessed on the wider side. Lio concluded that septoplasty leads to an improvement in the nasal performance on the afflicted side and no special affliction on the healthy side. However, in Dadgarnia et al study, according to the results from acoustic rhinometry, there was an increase in the nasal cross-sectional area on both the narrow and wide sides after the operation $(p<0.05)$, although this increase was not so notable in the narrower side after using decongestants. However, after the use of decongestants, a significant decrease in general nasal resistance through a rhinomanometry test was observed following surgery compared with presurgical values. These suggest importance of medical management in terms of use of decongestants for improvement of symptom of nose block.

The subjective feeling of nasal patency should ideally correlate with the objective measurements of nasal function; many have tested this association hypothesis. Some studies have used static objective tests, e.g., acoustic rhinometry, ${ }^{3,6-8}$ while others have used dynamic tests, e.g., 
rhinomanometry. ${ }^{7,9,10}$ Above tests require sophisticated and costly devices. It may require training to the person, a proper setup, and sufficient time duration for the test. Also, these instruments require regular calibration and maintenance. In our large government hospital (tertiary care center) with very heavy patient workload and absence of technical staff, we would prefer a simple-toperform, reasonably accurate, noninvasive, inexpensive method for objective evaluation of nasal patency - cold spatula test, which only requires a stainless steel spatula with calibrated markings that fulfils these criteria to a large extent. Care was taken to avoid heating of the metal plate when handling the test instrument and upon collecting the second expiration. However, it is not possible with this test instrument to fixate the condensation on the spatula, which rapidly disappears and is therefore highly dependent on the operator. Furthermore, airflow is dynamic, which affects the nasal condensation area. Finally, the spatula does not measure nasal pressure. Care was also taken during the cold spatula test that the subjects were seated in a correct position, their head orthostatic, and the placement of the metal plate centralized and horizontal. This positioning is still subject to tilting errors.

\section{CONCLUSION}

After review, we found that the literature has very few studies on the cold spatula test. There are no reference values, standardization data, or proof of efficiency. The cold spatula test offers objective evidence of the presence of nasal block and helps the clinician decide the veracity of the patient's complaint of nose block, which is highly subjective. This evidence helps the clinician continue or change the treatment offered for nose block instead of solely relying on the patient's subjective feeling. It, however, will not indicate the cause for nasal block for which DNE is necessary.

We conclude that:

- The air blast in cold spatula test is correlated well with subjective sensation of nose block, which was found to be statistically significant.
- Cold spatula test is a simple, reliable, easy-to-perform, and noninvasive objective test in investigating cases of nose block.

- All patients responded well to the necessary treatment (surgical or medical). This was documented by improvement in percentage in subjective sensation of nose block and also evident on cold spatula test as gradual increase in mean air blast on follow-up visits. This positive correlation was statistically significant.

\section{REFERENCES}

1. Brescovici S, Roithmann R. Modified Glatzel mirror test reproducibility in the evaluation of nasal patency. Rev Bras Otorrinolaringol 2008 Mar-Apr;74(2):215-222.

2. Hox V, Callebaut I, Bobic S, Jorissen M, Hellings PW. Nasal obstruction and smell impairment in nasal polyp disease: correlation between objective and subjective parameters. Rhinology 2010 Dec;48(4):426-432.

3. Melo DL, Santos RVM, Perilo TVC, Becker HMG, Motta AR. Mouth breathing evaluation: use of Glatzel mirror and peak nasal inspiratory flow. CoDAS 2013 Jan;25(3):236-241.

4. Gulati SP, Sachdeva OP, Wadhera R, Sodhi N, Garg A. Role of rhinomanometry to assess nasal airflow and resistance in patients undergoing septoplasty. Indian J Otolaryngol Head Neck Surg 2008 Apr-Jun;60(2):133-136.

5. Lio HJ. Outcome after nasal septoplasty. Otolaryngol Clin North Am 2010;52:205-221.

6. Dadgarnia MH, Baradaranfar MH, Mazidi M, Azimi meibodi SMR. Assessment of septoplasty effectiveness using acoustic rhinometry and rhinomanometry. Iran J Otorhinolaryngol 2013 Spring;25(2):71-78.

7. Mendes AIS, Wandalsen GF, Sole D. Objective and subjective assessments of nasal obstruction in children and adolescents with allergic rhinitis. J Pediatr (Rio J) 2012 Sep-Oct;88(5): 389-395.

8. Kamal I. Objective assessment of nasal obstruction in snoring and obstructive sleep apnea patients: experience of a Police Authority Hospital. Ann Saudi Med 2002 May-Jul;22(3-4): 158-162.

9. Kim SJ, Choi JH, Kim EJ, Lee SK, Lee SH, Jun YJ, Kim JH, Miyazaki S, Shin C. A prospective population-based study of total nasal resistance in Korean subjects. Clin Exp Otorhinolaryngol 2012 Mar;5(1):39-43.

10. Yoon DW, Lee SK, Yi H, Hong JH, Soichiro M, Lee SW, Kim JY, Shin C. Total nasal resistance (TNR) among Sasang constitutional types: a population-based study in Korea. BMC Complement Altern Med 2013 Nov;13(1):302. 